\title{
Primordial black hole production in inflationary models of supergravity with a single chiral superfield
}

\author{
Tie-Jun Gao* \\ School of Physics and Optoelectronic Engineering, Xidian University, Xi'an 710071, China \\ Zong-Kuan Guo ${ }^{\dagger}$ \\ CAS Key Laboratory of Theoretical Physics, Institute of Theoretical Physics, \\ Chinese Academy of Sciences, P.O. Box 2735, Beijing 100190, China
}

(Received 26 June 2018; published 21 September 2018)

\begin{abstract}
We propose a double inflection points inflationary model in supergravity with a single chiral superfield. Such a model allows for the generation of primordial black holes (PBHs) at small scales, which can account for a significant fraction of dark matter. Moreover, in vacuum it is possible to give a small and adjustable supersymmetry breaking with a tiny cosmological constant.
\end{abstract}

DOI: 10.1103/PhysRevD.98.063526

\section{INTRODUCTION}

The nature of dark matter remains an open question in modern cosmology. One of the simplest explanations is assuming primordial black holes (PBHs) are a significant component of dark matter. Recently, the detection of gravitational waves from binary black hole mergers by the LIGO and VIRGO opens a new window to probe black hole physics [1-3] and to explore the nature of dark matter.

It is known that PBHs can arise from high peaks of the curvature power spectrum at small scales via gravitational collapse during the radiation era, which could constitute dark matter today. Such a peak in the power spectrum can be generated in a single-field inflation with an inflection point [4] instead of using inflationary models with multiple fields [5-9]. However, in [10,11], it is pointed out that close to the inflection point, the ultraslow-roll trajectory supersede the slow-roll one, and thus, the slow-roll approximations cannot be used. So more precise approximation is used in [12], or the Mukhanov-Sasaki (MS) equation is numerically solved [13]. Recently, some inflationary models with an inflection point have been proposed to produce PBHs. For example, Ref. [14] explores the possibility of forming PBHs in the critical Higgs inflation, where the near-inflection point is related to the critical value of the renormalization group equation running of both the Higgs

\footnotetext{
*tjgao@xidian.edu.cn

guozk@itp.ac.cn
}

Published by the American Physical Society under the terms of the Creative Commons Attribution 4.0 International license. Further distribution of this work must maintain attribution to the author(s) and the published article's title, journal citation, and DOI. Funded by SCOAP ${ }^{3}$. self-coupling $\lambda$ and its nonminimal coupling to gravity $\xi$. And Ref. [15] argues that diffusion could induce an enhancement of the power spectrum. In [16] the author presents a toy model with a polynomial potential. PBHs product in Starobinsky's supergravity is presented in Ref. [17] where the scalar belongs to the vector multiplet. Reference [18] discusses the PBHs product in inflationary $\alpha$-attractors. Reference [19] provides a method to reconstruct the inflation potential from a given power spectrum, and gets a polynomial potential. In [20] the authors present a single-field inflationary model in string theory which allows for the generation of PBHs.

Although cosmological inflation is now established by all precise observational data such as the WMAP [21] and Planck data [22], the nature of inflation is still unknown. An interesting framework of inflation models building is to embed inflationary models into a more fundamental theory of quantum gravity, such as supergravity [23-25]. However, in the supergravity based inflationary models, it always suffers from the so-called $\eta$ problem [26]. The F-term of the potential is proportional to $e^{|\Phi|^{2}}$, which gives a contribution to the slow-roll parameter $\eta$ and breaks the slow-roll condition. One way to overcome such obstacles is to invoke a shift symmetry of the Kähler potential, and add an extra chiral superfield, which can be stabilized at the origin during inflation [27,28]. Another way is using a shiftsymmetric quartic stabilization term in the Kähler potential instead of a stabilizer superfield [29,30], and supersymmetry (SUSY) tends to be broken at a scale comparable to the inflation scale in such kinds of models. For instance, by using logarithmic Kähler potential and cubic superpotential, Ref. [31] constructs an inflection point inflationary model which have a non-SUSY de-Sitter vacuum responsible for the recent acceleration of the Universe. Whether SUSY is 
restored after inflation is quite important and worth discussing. Reference [32] point out if SUSY breaks at a scale higher than the intermediate scale, the electroweak vacuum may be unstable, in addition, the related paper [33] investigate this issue in detail, and find that the high-scale SUSY is still compatible with the known Higgs mass, though in a rather limited part of the parameter space. Therefore, the authors investigate the SUSY breaking properties of the model in Ref. [30] and study the conditions to restore SUSY after inflation.

In this paper, we shall consider the possibility to construct an inflection point inflationary model in supergravity with a single chiral superfield and focus on a superpotential with a sum of exponentials. We first assume SUSY restores after inflation, by fine-tuning the parameters of the model, such a superpotential can give a scalar potential with double inflection points. One of the inflection points can make the prediction of scalar spectral index and tensor-to-scalar ratio consistent with the current CMB constraints at large scales. The other inflection point can generate a large peak of the power spectrum at small scales to arise PBHs. After inflation, one can obtain small SUSY breaking and a tiny cosmological constant by introducing a nilpotent superfield $S$ $[30,34,35]$.

The paper is organized as follows. In the next section, we setup the inflection point inflationary model in supergravity with SUSY restoration after inflation. In Sec. III, we investigate the inflaton dynamics and compute the spectrum of primordial curvature perturbations of the model. In Sec. IV, we describe the mechanism of PBHs generation and calculate the mass distribution and abundance of PBHs. In Sec. V, we consider the possibility to get a small cosmological constant with SUSY breaking. The last section is devoted to summary.

\section{INFLECTION POINT INFLATION IN SUPERGRAVITY WITH SUSY RESTORED AFTER INFLATION}

In this section, we setup the inflection point inflationary model in supergravity and assumed that SUSY restores after inflation. The issue of SUSY breaking in vacuum is discussed in Sec. V.

Following Ref. [30], we consider a shift-symmetric Kähler potential of the form

$$
K=i c(\Phi-\bar{\Phi})-\frac{1}{2}(\Phi-\bar{\Phi})^{2}-\frac{\zeta}{4}(\Phi-\bar{\Phi})^{4},
$$

with $c$ and $\zeta$ are real constants. The real component $\phi$ of the chiral superfield $\Phi=(\phi+i \chi) / \sqrt{2}$ is taken to be the inflaton and the quartic term serves to stabilize the field $\phi$ during inflation at $\langle\chi\rangle \simeq 0$ by making $\zeta$ sufficient large.

The scalar potential is determined by a given superpotential $W$ as well as Kähler potential, which is given by

$$
V=e^{K / M_{P}^{2}}\left[D_{\Phi_{i}} W\left(K^{-1}\right)^{i j^{*}} D_{\Phi_{j}^{*}} W^{*}-3 M_{P}^{-2}|W|^{2}\right],
$$

where

$$
D_{\Phi} W=\partial_{\Phi} W+M_{P}^{-2}\left(\partial_{\Phi} K\right) W
$$

and $\left(K^{-1}\right)^{i j^{*}}$ is the inverse of the Kähler metric

$$
K^{i j^{*}}=\frac{\partial^{2} K}{\partial \Phi_{i} \partial \Phi_{j}^{*}} .
$$

In some inflationary models favored by the CMB data, the scalar potential can be generated by a superpotential which can be expanded as [30,36],

$$
W=\sum_{n \geq 0} a_{n} e^{-b_{n} \Phi},
$$

where $a_{n}$ and $b_{n}$ are constants. If we require the SUSY preservation in vacuum with a vanishing cosmological constant, the F-term should be vanished $D_{\Phi} W=0$, and $V=0$ at $\Phi=0$, which requires the constraint

$$
W=\partial_{\Phi} W=0 .
$$

In order to produce a significant fraction of PBHs from primordial density perturbations which are consistent with the cosmic microwave background (CMB) constraints, we consider a superpotential of the form

$$
W=a_{0}\left(1+a_{1} e^{-b_{1} \Phi}+a_{2} e^{-b_{2} \Phi}+a_{3} e^{-b_{3} \Phi}\right) .
$$

Such kinds of superpotential with exponential functions with two terms have been studied in the so-called racetrack model [36-38] and in other models [30]. By solving the constraint (6), we can eliminate two of the parameters $a_{1}$ and $a_{2}$ as

$a_{1} \rightarrow \frac{b_{2}+a_{3} b_{2}-a_{3} b_{3}}{b_{1}-b_{2}}, \quad a_{2} \rightarrow \frac{-b_{1}-a_{3} b_{1}+a_{3} b_{3}}{b_{1}-b_{2}}$.

Substituting the superpotential (7) and Kähler potential (1) into (2), one can get the scalar potential $V(\phi)$. In order to make the scalar potential predicted the primordial spectra consistent with the $\mathrm{CMB}$ data, give enough e-folding numbers and produce a significant fraction of $\mathrm{PBHs}$ in an interesting window for dark matter, we fine-tune the model parameters, and find some range in parameter space. We list two examples of parameter sets in Table I, and the

TABLE I. Examples of parameter choice.

\begin{tabular}{ccccccc}
\hline \hline & $a_{0}$ & $a_{3}$ & $b_{1}$ & \multicolumn{1}{c}{$b_{2}$} & $b_{3}$ & $c$ \\
\hline 1 & $4.35 \times 10^{-6}$ & $7 \times 10^{-8}$ & 3.05 & 6.3868164 & -4.4 & 2.8 \\
2 & $4.06 \times 10^{-6}$ & $1 \times 10^{-6}$ & 2.89 & 7.251197 & -3.2 & 2.85 \\
\hline \hline
\end{tabular}




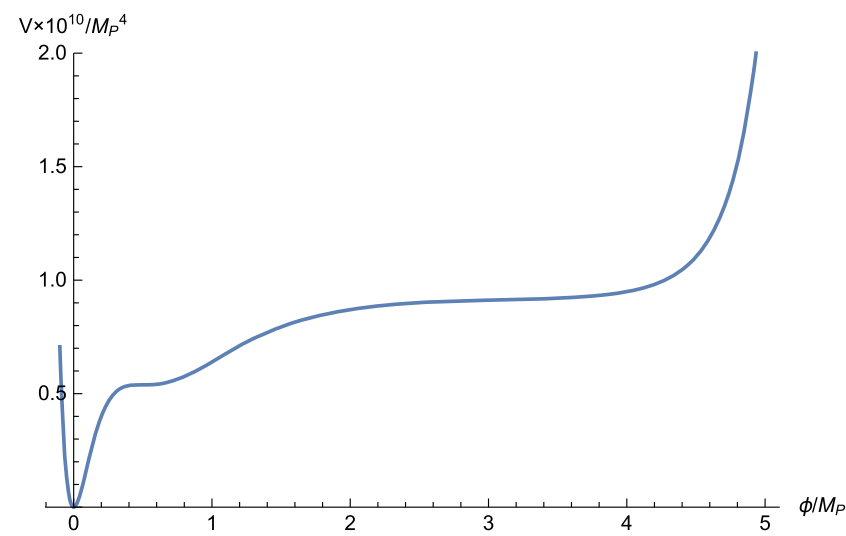

FIG. 1. Scalar potential $V(\phi)$ for the first parameter set of Table I.

scalar potential $V(\phi)$ for the first parameter set is depicted in Fig. 1.

We can see that the potential have two nearly inflection points, one of the inflection points can make the prediction of scalar spectral index and tensor-to-scalar ratio consistent with the current CMB data, and the other one at small scales can generate a large peak in the power spectrum to arise PBHs. We will discuss these issues in the following sections.

\section{THE SPECTRUM OF PRIMORDIAL CURVATURE PERTURBATIONS}

In the Friedmann-Robertson-Walker homogeneous background, the Friedmann equation and the inflaton field equation can be written as

$$
\begin{gathered}
H^{2}=\frac{1}{3 M_{P}^{2}}\left(\frac{1}{2} \dot{\phi}^{2}+V(\phi)\right), \\
\ddot{\phi}+3 H \dot{\phi}+V^{\prime}(\phi)=0,
\end{gathered}
$$

where dots represent derivatives with respect to cosmic time and primes denote derivatives with respect to the field $\phi$. The e-folding numbers from an initial time $t_{i}$ is defined as

$$
N_{e}(t)=\int_{t_{i}}^{t} H(t) d t
$$

where the e-folding number between the crossing time at the scale of $k_{0.001}$ and the time of the inflation end is required in the range 50-60. In this paper, we use $\Delta N_{e}^{*}$ to denote the e-folding number between $k_{*}=k_{0.05}$ and the end of inflation, which should be in the range 45-55.

In the single-field slow-roll framework, the slow-roll parameters $\epsilon$ and $\eta$ can be calculated as

$$
\begin{aligned}
& \epsilon=\frac{M_{P}^{2}}{2}\left(\frac{V^{\prime}}{V}\right)^{2}, \\
& \eta=M_{P}^{2}\left(\frac{V^{\prime \prime}}{V}\right) .
\end{aligned}
$$

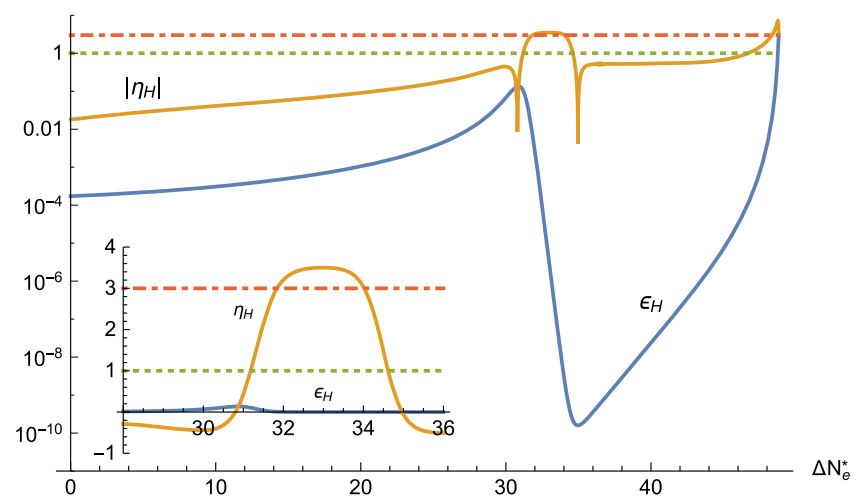

FIG. 2. Slow-roll parameters $\epsilon_{H}$ (blue solid line) and $\eta_{H}$ (orange solid line) as functions of the e-folding number $\Delta N_{e}^{*}$ for parameter set 1 . The green dashed line and red dot-dash line indicate the values 1 and 3 respectively. The inset zooms into the relevant range of $\Delta N_{e}^{*}$ for the ultraslow-roll inflation where the slow-roll approximation fails.

However, in Refs. $[10,11]$ it is pointed out that close to the inflection point, the ultraslow-roll trajectory supersedes the slow-roll one and thus one should use the slow-roll parameters defined by the Hubble parameter [39-41],

$$
\begin{aligned}
\epsilon_{H} & =-\frac{\dot{H}}{H^{2}}, \\
\eta_{H} & =-\frac{\ddot{H}}{2 H \dot{H}}=\epsilon_{H}-\frac{1}{2} \frac{d \ln \epsilon_{H}}{d N_{e}}, \\
\xi_{H} & =\frac{H}{2 H^{2} \dot{H}}-2 \eta_{H}^{2}=\epsilon_{H} \eta_{H}-\frac{d \eta_{H}}{d N_{e}} .
\end{aligned}
$$

Since near the inflection point, the potential becomes extremely flat, so the slope term $V^{\prime}(\phi)$ of Eq. (10) may reduce drastically, which means $\left|\eta_{H}\right|=|-\ddot{\phi} / H \dot{\phi}| \simeq 3$. For a nearly inflection point there exist a range satisfies $\ddot{\phi}+3 H \dot{\phi}=-V^{\prime}(\phi)>0$ and $\dot{\phi}<0$, which leads to $\left|\eta_{H}\right|>3$,

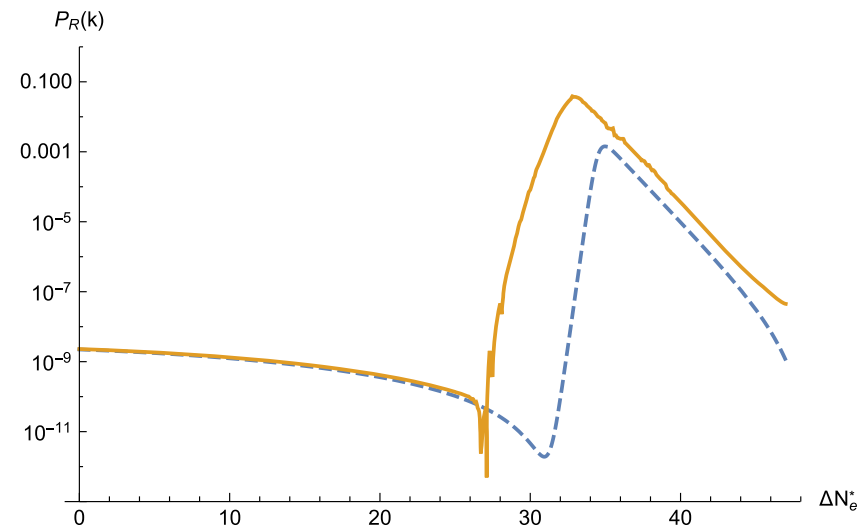

FIG. 3. Primordial power spectrum as a function of the e-folding numbers $\Delta N_{e}^{*}$ for parameter set 1 . The blue solid line is calculated by using the MS equation and the orange dashed line is calculated by using the approximation (15). 
TABLE II. Results of inflationary observables at CMB and small scales.

\begin{tabular}{cccccccc}
\hline \hline & $n_{s}$ & $r$ & $\alpha$ & $\Delta N_{e}^{*}$ & $P_{R}^{\text {peak }}$ & $M_{\mathrm{PBHs}}^{\text {peak }} / M_{\odot}$ & $\Omega_{\mathrm{PBH}} / \Omega_{\mathrm{DM}}$ \\
\hline 1 & 0.9635 & 0.00276 & -0.00369 & 48.66 & 0.0369 & $5.50 \times 10^{-14}$ & 0.114 \\
2 & 0.9649 & 0.00257 & -0.00286 & 46.29 & 0.0032 & $1.39 \times 10^{-16}$ & 0.022 \\
\hline \hline
\end{tabular}

thus the slow-roll approximation is no longer applicable. The Hubble slow-roll parameters $\epsilon_{H}$ and $\eta_{H}$ as functions of the e-folding number $\Delta N_{e}^{*}$ for parameter set 1 are show in Fig. 2.

We can see that near the inflection point the slow-roll parameter $\left|\eta_{H}\right|>3$, so the slow-roll approximation fails. There is a valley on the curve of $\epsilon_{H}$, which can give rise to a large peak of the primordial power spectrum.

At the leading order, the scalar spectral index and its running as well as the tensor-to-scalar ratio can be expressed using $\epsilon_{H}, \eta_{H}$ and $\xi_{H}$ as

$$
\begin{aligned}
n_{s} & =1-4 \epsilon_{H}+2 \eta_{H}, \\
\alpha & =\frac{d n_{s}}{d \ln k}=10 \epsilon_{H} \eta_{H}-8 \epsilon_{H}^{2}-2 \xi_{H}, \\
r & =16 \epsilon_{H} .
\end{aligned}
$$

Then the scalar power spectrum can be approximately calculated using the expression

$$
P_{R} \simeq \frac{1}{8 \pi^{2} M_{P}^{2}} \frac{H^{2}}{\epsilon_{H}} .
$$

The observational constraints from Planck on the scalar spectral index $n_{s}$ and its running $\alpha$, the tensor-to-scalar ratio $r$ and amplitude of the primordial curvature perturbations $A_{s}$ at $68 \%$ C.L. at a scale $k_{*}=0.05 \mathrm{Mpc}^{-1}$ are [22]

$$
\begin{aligned}
n_{s} & =0.9650 \pm 0.0050, \\
\alpha & =-0.009 \pm 0.008, \\
r & <0.07, \\
A_{s} & =2.2 \pm 0.1 \times 10^{-9} .
\end{aligned}
$$

However, in order to compute the power spectrum near the inflection point more reliably, one must solve the MS equation of mode function

$$
\frac{d^{2} u_{k}}{d \tau^{2}}+\left(k^{2}-\frac{1}{z} \frac{d^{2} z}{d \tau^{2}}\right) u_{k}=0
$$

where $\tau$ denotes conformal time and $z \equiv \frac{a}{\mathcal{H}} \frac{d \phi}{d \tau}$. The initial condition for Eq. (17) is taken to be the Bunch-Davies type [42]

$$
u_{k} \rightarrow \frac{e^{-i k \tau}}{\sqrt{2 k}}, \quad \text { as } \frac{k}{a H} \rightarrow \infty .
$$

For the purpose of numerical simulation, the MS equation can be written in terms of $N_{e}$ as the time variable [13]

$$
\begin{aligned}
& \frac{d^{2} u_{k}}{d N_{e}^{2}}+\left(1-\epsilon_{H}\right) \frac{d u_{k}}{d N_{e}}+\left[\frac{k^{2}}{\mathcal{H}^{2}}+\left(1+\epsilon_{H}-\eta_{H}\right)\left(\eta_{H}-2\right)\right. \\
& \left.-\frac{d \epsilon_{H}-\eta_{H}}{d N_{e}}\right]=0,
\end{aligned}
$$

and the power spectrum can be calculated by

$$
\mathcal{P}_{\mathcal{R}}=\frac{k^{3}}{2 \pi^{2}}\left|\frac{u_{k}}{z}\right|_{k \ll \mathcal{H}}^{2} .
$$

In Fig. 3, we plot the primordial power spectrum as a function of the e-folding number $\Delta N_{e}^{*}$ with parameter set 1 . The solid line is calculated from the solutions to the MS equation, while the dashed line is calculated by using the approximation (15), which underestimates the power spectrum, thus couldn't be used to obtain the PBH abundance and mass. We can see that there is a large peak at small scales, with a height of about seven orders of magnitude more than the spectrum at CMB scales, which can generate PBHs via gravitational collapse.

Our numerical results of inflationary dynamics corresponding to the two parameter sets are presented in Table II, and they are in agreement with the current CMB constraints on the primordial spectra (16).

\section{PRODUCTION OF PRIMORDIAL BLACK HOLES}

The mechanisms of $\mathrm{PBHs}$ production have been studies in several Refs. [43-47]. And in this section, we will calculate the PBH abundance using the Press-Schechter approach [48] of gravitational collapse. When a large amplitude of primordial fluctuations, which is generated at small scales during inflation and reenters the Hubble horizon after inflation. It undergoes gravitational collapse and form PBHs if the density fluctuation of matter is significantly large.

The mass of the resulting PBHs is assumed to be directly proportional to the horizon mass at reentry time,

$$
M=\gamma M_{H}=\gamma \frac{4}{3} \pi \rho H^{-3} .
$$

It can be approximated as [13]

$M \simeq 10^{18} \mathrm{~g}\left(\frac{\gamma}{0.2}\right)\left(\frac{g_{*}}{106.75}\right)^{-1 / 6}\left(\frac{k}{7 \times 10^{13} \mathrm{Mpc}^{-1}}\right)^{-2}$, 


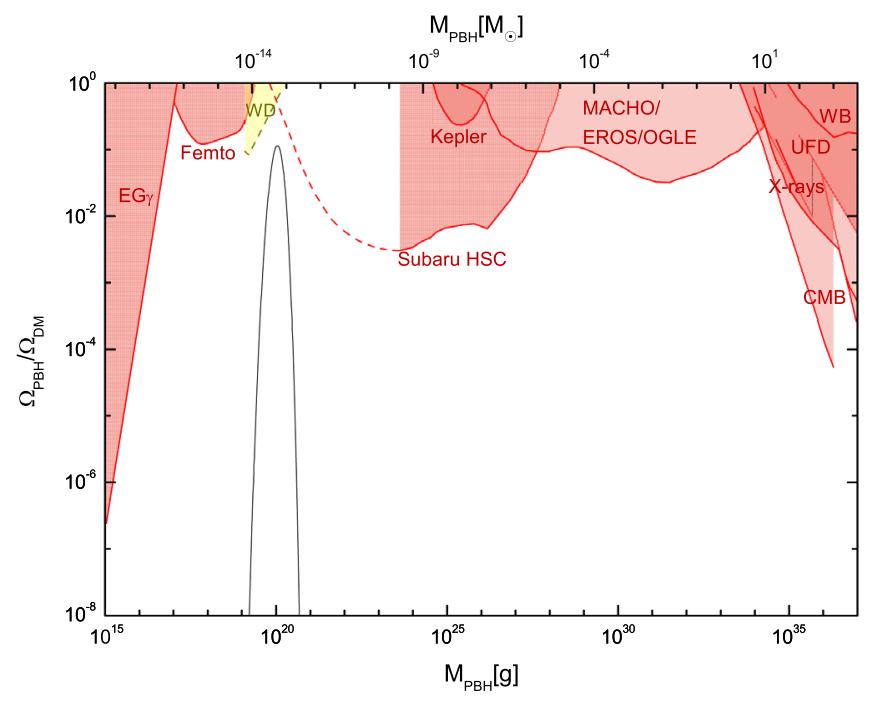

FIG. 4. The factional abundance of PBHs for the first parameters set in Table I and the observational constraints including the extragalactic gamma-rays from the $\mathrm{PBH}$ evaporation (EG $\gamma$ ) [55], the femtolensing of gamma-ray bursts(Femto) [56], the white dwarfs explosion (WD) [57], the microlensing events with Subaru HSC (Subaru HSC) [58] (The red dotted line shows the uncertain constraint of HSC), with Kepler satellite (Kepler) [59], with MACHO/EROS/OGLE [60-62], the CMB measurements $[63,64]$, the radio and x-ray observations $[65,66]$, the dynamical heating of dwarf galaxies and ultrafaint dwarf galaxies (UFD) [67,68] and the distribution of wide binaries (WB) [69].

where $\gamma \sim 0.2$ is a proportionality constant, which depends on the details of the gravitational collapse [49], and $g_{*} \sim$ 106.75 is the effective degrees of freedom for energy density, which is equal to that of entropy density.

In the context of the Press-Schechter model of gravitational collapse, assuming that the probability distribution of density perturbations is Gaussian with width $\sigma(M)$, the formation rate of PBHs, which we denote as $\beta(M)$ is given by

$$
\begin{aligned}
\beta(M) & \equiv \frac{\rho_{\mathrm{PBH}}(M)}{\rho_{\mathrm{tot}}}=\frac{1}{\sqrt{2 \pi \sigma^{2}(M)}} \int_{\delta_{c}}^{\infty} d \delta \exp \left(\frac{-\delta^{2}}{2 \sigma^{2}(M)}\right) \\
& =\frac{1}{2} \operatorname{erfc}\left(\frac{\delta_{c}}{\sqrt{2 \sigma^{2}(M)}}\right)
\end{aligned}
$$

where $\delta_{c} \simeq 0.45[50,51]$ denotes the threshold of density perturbations for the collapse into PBHs. Here $\sigma^{2}(M)$ is the variance of the comoving density perturbations coarse grained at a scale $R=1 / k$, during the radiation-dominated era, which is given by,

$$
\sigma^{2}(M(k))=\frac{16}{81} \int \frac{d q}{q}(q R)^{4} P_{R}(q) W(q R)^{2},
$$

where $P_{R}$ is the power spectrum of the primordial comoving curvature perturbations, and the smoothing window function $W(x)$ is taken to be a Gaussian $W(x)=$ $\exp \left(-x^{2} / 2\right)$. Then integrating over all masses $M$ one can get the present abundance of PBHs,

$$
\Omega_{\mathrm{PBH}}=\int \frac{d M}{M} \Omega_{\mathrm{PBH}}(M),
$$

with

$$
\begin{aligned}
\frac{\Omega_{\mathrm{PBH}}(M)}{\Omega_{\mathrm{DM}}}= & \left(\frac{\beta(M)}{1.6 \times 10^{-16}}\right)\left(\frac{\gamma}{0.2}\right)^{3 / 2}\left(\frac{g_{*}}{106.75}\right)^{-1 / 4} \\
& \times\left(\frac{M}{10^{18} \mathrm{~g}}\right)^{-1 / 2}
\end{aligned}
$$

where $\Omega_{\mathrm{DM}} \simeq 0.26$ is the total dark matter abundance [52].

In Fig. 4, we plot the factional abundance of PBHs for the first parameter set in Table I and the observational constraints from Refs. $[53,54]$ The numerical results of the PBHs mass and abundance for the two parameters sets are listed in Table II.

\section{SUSY BREAKING AND VACUUM STRUCTURE}

After inflation, in order to obtain small SUSY breaking and a very small cosmological constant, it is possible to extend the Kähler potential and superpotential with a nilpotent superfield $S[34,35]$. Since such a method is discussed in Ref. [30], we just list some conclusions.

The Kähler potential and superpotential can be extended as

$$
\begin{aligned}
K & =K^{\text {inf }}+S \bar{S}, \\
W & =W^{\text {inf }}+W_{0}+\mu^{2} S,
\end{aligned}
$$

where $W_{0}$ and $\mu$ are constants and the upper indexes denote the quantities in the inflation sector as in Eqs. (1) and (7). In the leading order of $\left|W_{0}\right|$ and $|\mu|^{2}$, the vacuum expectation value of the inflation is

$$
\langle\Phi\rangle=-\frac{W_{0} K_{\Phi}^{\mathrm{inf}}}{W_{\Phi \Phi}^{\mathrm{inf}}} .
$$

Then the vacuum energy becomes

$$
V=|\mu|^{4}-3\left|W_{0}\right|^{2}
$$

Since the SUSY breaking scale $\left|D_{S} W\right|=|\mu|^{2}$ can be chosen freely, by fine-tuning the parameters one can get a small and adjustable cosmological constant of order $10^{-120}$ responsible for the current accelerated expansion of the Universe.

The role of the nilpotent superfield in this work just leads to a tiny cosmological constant, and cannot be used for large resolution of masses between known particles and their superpartners in particles physics. In addition, nilpotent 
superfields and related nonlinear realizations are not necessary for SUSY breaking. Other models having linearly realizations and spontaneously SUSY breaking are discussed in [70] with a single vector (inflaton) superfield instead of a single chiral inflaton superfield.

\section{SUMMARY}

In this paper, we propose a double inflection point inflationary model in supergravity with a single chiral superfield. We focus on a superpotential with a sum of exponentials and assume the SUSY restores after inflation. We find that such a superpotential can give a scalar potential with double inflection points. We investigate the inflaton dynamics and compute the spectrum of primordial curvature perturbations, and find that the inflection point at large scales can make the predicted of scalar spectral index and tensor-to-scalar ratio consistent with the current $\mathrm{CMB}$ data.

The other inflection point at small scales can generate a large peak in the power spectrum with a height of about seven orders of magnitude more than the spectrum at CMB scales. When the significantly large amplitude of primordial fluctuation reenters the Hubble horizon after inflation, it will undergo gravitational collapse and form PBHs. Moreover, we describe the mechanism of PBHs generation, and get the mass and abundance of PBHs which can account for a significant component of dark matter.

After inflation, it is possible to give a small and adjustable SUSY breaking and a tiny cosmological constant by extending the Kähler potential and superpotential with a nilpotent superfield. Since the SUSY breaking scale is much lower than the inflation scale, the effects of nilpotent superfield $S$ on the inflationary dynamics can be negligible.

\section{ACKNOWLEDGMENTS}

This work was supported by "the National Natural Science Foundation of China" (NNSFC) with Grant No. 11705133, and "the Fundamental Research Funds for the Central Universities" No. JBF180501. Z. K. G. is supported in part by the National Natural Science Foundation of China Grants No. 11575272, No. 11690021, and No. 11335012.
[1] B. P. Abbott et al., Observation of Gravitational Waves from a Binary Black Hole Merger, Phys. Rev. Lett. 116, 061102 (2016).

[2] B. P. Abbott et al., GW151226: Observation of Gravitational Waves from a 22-Solar-Mass Binary Black Hole Coalescence, Phys. Rev. Lett. 116, 241103 (2016).

[3] B. P. Abbott et al., GW170104: Observation of a 50-SolarMass Binary Black Hole Coalescence at Redshift 0.2, Phys. Rev. Lett. 118, 221101 (2017).

[4] J. Garcia-Bellido and E. R. Morales, Primordial black holes from single field models of inflation, Phys. Dark Universe 18, 47 (2017).

[5] J. Garcia-Bellido, A. D. Linde, and D. Wands, Density perturbations and black hole formation in hybrid inflation, Phys. Rev. D 54, 6040 (1996).

[6] J. Yokoyama, Formation of MACHO primordial black holes in inflationary cosmology, Astron. Astrophys. 318, 673 (1997).

[7] T. Nakamura, M. Sasaki, T. Tanaka, and K. S. Thorne, Gravitational waves from coalescing black hole MACHO binaries, Astrophys. J. 487, L139 (1997).

[8] S. Clesse and J. Garca-Bellido, Massive primordial black holes from hybrid inflation as dark matter and the seeds of galaxies, Phys. Rev. D 92, 023524 (2015).

[9] J. Garcia-Bellido, M. Peloso, and C. Unal, Gravitational waves at interferometer scales and primordial black holes in axion inflation, J. Cosmol. Astropart. Phys. 12 (2016) 031.
[10] C. Germani and T. Prokopec, On primordial black holes from an inflection point, Phys. Dark Universe 18, 6 (2017).

[11] K. Dimopoulos, Ultra slow-roll inflation demystified., Phys. Lett. B 775, 262 (2017).

[12] H. Motohashi and W. Hu, Primordial black holes and slowroll violation, Phys. Rev. D 96, 063503 (2017).

[13] G. Ballesteros and M. Taoso, Primordial black hole dark matter from single field inflation, Phys. Rev. D 97, 023501 (2018).

[14] J. M. Ezquiaga, J. Garcia-Bellido, and E. R. Morales, Primordial black hole production in critical Higgs inflation, Phys. Lett. B 776, 345 (2018).

[15] J. M. Ezquiaga and J. Garcia-Bellido, Quantum diffusion beyond slow-roll: implications for primordial black-hole production, J. Cosmol. Astropart. Phys. 08 (2018) 018.

[16] Y. Gong, Primordial black holes from ultra-slow-roll inflation, J. Cosmol. Astropart. Phys. 07 (2018) 007.

[17] A. Addazi, A. Marciano, S. V. Ketov, and M. Y. Khlopov, Physics of superheavy dark matter in supergravity, Int. J. Mod. Phys. D 27, 1841011 (2018).

[18] I. Dalianis, A. Kehagias, and G. Tringas, Primordial black holes from $\alpha$-attractors, arXiv:1805.09483.

[19] M. P. Hertzberg and M. Yamada, Primordial black holes from polynomial potentials in single field inflation, Phys. Rev. D 97, 083509 (2018).

[20] M. Cicoli, V. A. Diaz, and F. G. Pedro, Primordial black holes from string inflation, J. Cosmol. Astropart. Phys. 06 (2018) 034 . 
[21] G. Hinshaw et al. (WMAP Collaboration), Nine-year Wilkinson Microwave Anisotropy Probe (WMAP) observations: Cosmological parameter results, Astrophys. J. Suppl. Ser. 208, 19 (2013).

[22] P. A. R. Ade et al., Planck 2015 results. XX. Constraints on inflation., Astron. Astrophys. 594, A20 (2016).

[23] D. Z. Freedman, P. van Nieuwenhuizen, and S. Ferrara, Progress toward a theory of supergravity, Phys. Rev. D 13, 3214 (1976).

[24] S. Deser and B. Zumino, Consistent supergravity, Phys. Lett. B 62, 335 (1976).

[25] J. Wess and J. Bagger, Supersymmetry and Supergravity, 2nd ed. (Princeton University Press, Princeton, New Jersey, 1992).

[26] M. Yamaguchi, Supergravity based inflation models: A review, Classical Quantum Gravity 28, 103001 (2011).

[27] M. Kawasaki, M. Yamaguchi, and T. Yanagida, Natural Chaotic Inflation in Supergravity, Phys. Rev. Lett. 85, 3572 (2000).

[28] M. Kawasaki, M. Yamaguchi, and T. Yanagida, Natural chaotic inflation in supergravity and leptogenesis, Phys. Rev. D 63, 103514 (2001).

[29] S. V. Ketov and T. Terada, Inflation in supergravity with a single chiral superfield, Phys. Lett. B 736, 272 (2014).

[30] S. V. Ketov and T. Terada, On SUSY restoration in singlesuperfield inflationary models of supergravity, Eur. Phys. J. C 76, 438 (2016).

[31] T.-J. Gao and Z.-K. Guo, Inflection point inflation and dark energy in supergravity, Phys. Rev. D 91, 123502 (2015).

[32] G. Degrassi, S. Di Vita, J. Elias-Miro, J. R. Espinosa, G. F. Giudice, G. Isidori, and A. Strumia, Higgs mass and vacuum stability in the standard model at NNLO, J. High Energy Phys. 08 (2012) 098.

[33] G. F. Giudice and A. Strumia, Probing high-scale and split supersymmetry with Higgs mass measurements, Nucl. Phys. B858, 63 (2012).

[34] S. Ferrara, R. Kallosh, and A. Linde, Cosmology with nilpotent superfields, J. High Energy Phys. 10 (2014) 143.

[35] R. Kallosh and A. Linde, Inflation and uplifting with nilpotent superfields, J. Cosmol. Astropart. Phys. 01 (2015) 025 .

[36] J. J. Blanco-Pillado, C. P. Burgess, J. M. Cline, C. Escoda, M. Gomez-Reino, R. Kallosh, A. Linde, and F. Quevedo, Racetrack inflation., J. High Energy Phys. 11 (2004) 063.

[37] C. Escoda, M. Gomez-Reino, and F. Quevedo, Saltatory de Sitter string vacua, J. High Energy Phys. 11 (2003) 065.

[38] N. V. Krasnikov, On supersymmetry breaking in superstring theories, Phys. Lett. B 193, 37 (1987).

[39] D. J. Schwarz, C. A. Terrero-Escalante, and A. A. Garcia, Higher order corrections to primordial spectra from cosmological inflation, Phys. Lett. B 517, 243 (2001).

[40] S. M. Leach, A. R. Liddle, J. Martin, and D. J Schwarz, Cosmological parameter estimation and the inflationary cosmology, Phys. Rev. D 66, 023515 (2002).

[41] D. J. Schwarz and C. A. Terrero-Escalante, Primordial fluctuations and cosmological inflation after WMAP 1.0, J. Cosmol. Astropart. Phys. 08 (2004) 003.

[42] T. S. Bunch and P. C. W. Davies, Quantum field theory in de Sitter space: renormalization by point-splitting, Proc. R. Soc. A 360, 117 (1978).
[43] M. Y. Khlopov and A. G. Polnarev, Primordial black holes as a cosmological test of grand unification, Phys. Lett. 97B, 383 (1980).

[44] M. Y. Khlopov, R. V. Konoplich, S. G. Rubin, and A. S. Sakharov, First-order phase transitions as a source of black holes in the early universe, Grav. Cosmol. S 6, 1 (2000).

[45] M. Y. Khlopov, Primordial black holes, Res. Astron. Astrophys. 10, 495 (2010).

[46] K. M. Belotsky, A. D. Dmitriev, E. A. Esipova, V. A. Gani, A. V. Grobov, M. Y. Khlopov, A. A. Kirillov, S. G. Rubin, and I. V. Svadkovsky, Signatures of primordial black hole dark matter, Mod. Phys. Lett. A 29, 1440005 (2014).

[47] C. Germani and I. Musco, The abundance of primordial black holes depends on the shape of the inflationary power spectrum, arXiv:1805.04087.

[48] W. H. Press and P. Schechter, Formation of galaxies and clusters of galaxies by selfsimilar gravitational condensation, Astrophys. J. 187, 425 (1974).

[49] B. J. Carr, The primordial black hole mass spectrum, Astrophys. J. 201, 1 (1975).

[50] I. Musco and J. C. Miller, Primordial black hole formation in the early universe: Critical behaviour and self-similarity, Classical Quantum Gravity 30, 145009 (2013).

[51] T. Harada, C.-M. Yoo, and K. Kohri, Threshold of primordial black hole formation, Phys. Rev. D 88, 084051 (2013); Erratum, Phys. Rev. D89, 029903(E) (2014).

[52] P. A. R. Ade et al., Planck 2015 results. XIII. Cosmological parameters, Astron. Astrophys. 594, A13 (2016).

[53] K. Inomata, M. Kawasaki, K. Mukaida, and T. T. Yanagida, Double inflation as a single origin of primordial black holes for all dark matter and LIGO observations, Phys. Rev. D 97, 043514 (2018).

[54] S. Pi, Y.-1. Zhang, Q.-G. Huang, and M. Sasaki, Scalaron from $R^{2}$-gravity as a heavy field, J. Cosmol. Astropart. Phys. 05 (2018) 042.

[55] B. J. Carr, K. Kohri, Y. Sendouda, and J. Yokoyama, New cosmological constraints on primordial black holes, Phys. Rev. D 81, 104019 (2010).

[56] A. Barnacka, J. F. Glicenstein, and R. Moderski, New constraints on primordial black holes abundance from femtolensing of gamma-ray bursts, Phys. Rev. D 86, 043001 (2012).

[57] P. W. Graham, S. Rajendran, and J. Varela, Dark matter triggers of supernovae, Phys. Rev. D 92, 063007 (2015).

[58] H. Niikura, M. Takada, N. Yasuda, R. H. Lupton, T. Sumi, S. More, A. More, M. Oguri, and M. Chiba, Microlensing constraints on primordial black holes with the Subaru/HSC Andromeda observation, arXiv:1701.02151.

[59] K. Griest, A. M. Cieplak, and M. J. Lehner, New limits on primordial black hole dark matter from an analysis of Kepler source microlensing data, Phys. Rev. Lett. 111, 181302 (2013).

[60] R. A. Allsman et al. (Macho), MACHO Project Limits on Black Hole Dark Matter in the 1-30 M $\odot$ Range, Astrophys. J. 550, L169 (2001).

[61] P. Tisserand et al. (EROS-2), Limits on the Macho content of the Galactic Halo from the EROS-2 Survey of the Magellanic Clouds, Astron. Astrophys. 469, 387 (2007).

[62] L. Wyrzykowski et al., The OGLE view of microlensing towards the Magellanic Clouds - IV. OGLE-III SMC data 
and final conclusions on MACHOs, Mon. Not. R. Astron. Soc. 416, 2949 (2011).

[63] V. Poulin, P. D. Serpico, F. Calore, S. Clesse, and K. Kohri, CMB bounds on disk-accreting massive primordial black holes, Phys. Rev. D 96, 083524 (2017).

[64] Y. Ali-Haïmoud and M. Kamionkowski, Cosmic microwave background limits on accreting primordial black holes, Phys. Rev. D 95, 043534 (2017).

[65] Y. Inoue and A. Kusenko, New X-ray bound on density of primordial black holes, J. Cosmol. Astropart. Phys. 10 (2017) 034.

[66] D. Gaggero, G. Bertone, F. Calore, R. M. T. Connors, M. Lovell, S. Markoff, and E. Storm, Searching for Primordial
Black Holes in the Radio and X-Ray Sky, Phys. Rev. Lett. 118, 241101 (2017).

[67] S. M. Koushiappas and A. Loeb, Dynamics of Dwarf Galaxies Disfavor Stellar-Mass Black Holes as Dark Matter, Phys. Rev. Lett. 119, 041102 (2017).

[68] T.D. Brandt, Constraints on macho dark matter from compact stellar systems in ultra-faint dwarf galaxies, Astrophys. J. 824, L31 (2016).

[69] M. A. Monroy-Rodrguez and C. Allen, The end of the MACHO era, revisited: new limits on MACHO masses from halo wide binaries, Astrophys. J. 790, 159 (2014).

[70] A. Addazi, S. Ketov, and M. Khlopov, Gravitino and Polonyi production in supergravity, Eur. Phys. J. C 78, 642 (2018). 